\title{
EL VALOR COMO CUALIDAD RELACIONAL ${ }^{2}$
}

Evidently, however, none of these arguments are really decisive, and the position is extremely unsatisfactory to any one with real curiosity about such a fundamental question. In such cases it is a heuristic maxim that the truth lies not in one of the two disputed views but in some third possibility which has not yet been thought of, which we can only discover by rejecting something assumed as obvious by both the disputants.

(F. F. Ramsey, The Foundations of Mathematics, pp. 115116.)

El valor es la cualidad que adquiere lo deseable cuando es deseado en una situación determinada. No se puede identificar, como afirman los objetivistas, con la deseabilidad o, como proponen los subjetivistas con el deseo.

No es la deseabilidad, porque el ser deseable no implica necesariamente que lo deseable tenga valor en un momento dado o que sea valioso en la medida que sea deseable, pues podemos sin dificultad y con completo sentido preguntar, por ejemplo, si lo deseable tiene valor:

Todo lo que existe es en cierto modo dese-able, o sea, incluye, como lo muestra la composición de la palabra, la posibilidad de ser deseado en circunstancias propicias. Pero como posibilidad, la deseabilidad no es valor, ya que, propiamente dicho, no es algo que posee el objeto, sino sólo una posibilidad de valer o, como se diría en jerga aristotélica, un valor potencial. ${ }^{2}$ El diamante que yace oculto en una mina de Sudáfrica es, sin duda, dese-able, pero no siendo deseado, ya que no lo hemos visto nunca y no sabemos que existe ni si lo podríamos conseguir en caso de que existiese, carece de valor. ¿A

1 Quiero aclarar que uno de los supuestos de que depende la validez de este trabajo es la doctrina que acepta la inteligibilidad y existencia de cualidades relacionales. Si no hay cualidades relacionales, el valor no puede ser una de ellas. No discuto la doctrina aquí porque pertenece a la ontología, mientras que el presente trabajo cae dentro de la axiologia. Además, quiero agradecerle al Prof. R. Frondizi su gentileza al haber leído este ensayo y haber hecho varios comentarios y sugerencias de gran utilidad.

2 Esta distinción entre valor potencial y actual no es nueva. Ya Meinong la hacía en su Psychologische-ethische Untersuchungen zur Werttheorie (Graz, 1894), sin duda inspirado en la enseñanza de su maestro $F$. Brentano. Meinong, sin embargo, parece apartarse del pensamiento aristotélico ortodoxo al concebir la potencialidad como algo positivo en el objeto y no como mera privación, pues afirma que el valor potencial es el valor que el objeto posee cuando no está en la presencia del sujeto. Esto es inaceptable desde el punto de vista expuesto en este ensayo. El llamado "valor potencial" no es propiamente valor en un sentido ontológico que implique la posesión de alguna realidad o actualidad; es sólo la posibilidad de valer que el objeto tiene como objeto y que aquí identificamos con la deseabilidad. 
quién se le ocurriría venderlo y, menos aún, comprarlo en base a la mención que se ha hecho aquí de él? El valor, considerado como actual y vigente, incluye la determinación subjetiva, el deseo, por lo que no se puede identificar con la mera posibilidad de valer o deseabilidad. Nuestro diamante puede valer, pero no vale. Su valor es sólo una posibilidad actualizable por circunstancias propicias, una posibilidad múltiple y casi infinita sin embargo, gracias al gran número de circunstancias en que el objeto se podría encontrar. Lo podríamos imaginar como deseado no sólo para ponerlo en la sortija de nuestra amada en el momento de comprometernos, o para asegurar nuestro capital durante un periodo inflacionario peligroso, sino también, por ejemplo, como pisapapeles si es suficientemente pesado, para cortar un cristal, o, en fin, para todo aquello que su naturaleza concreta permita. ${ }^{3}$

Que el valor no pueda identificarse con la dese-a-bilidad quiere decir, entre otras cosas, que no es una cualidad absoluta, como lo es, por ejemplo, el tamaño en la oración " $x$ tiene dos metros de alto". Es más bien una cualidad relacional, producto de la relación sujeto-objeto en determinadas circunstancias y no perteneciente al objeto en sí, independientemente del sujeto. $\mathrm{Si}$ fuera una cualidad absoluta, las cosas tendrían valor aun sin su descubrimiento por un sujeto, como es el caso del tamaño en el ejemplo dado. Un vaso de agua, un diamante y una silla de madera, todos tendrían valor, $y$, más aún, el mismo valor, o sea, un valor constante, independiente del sujeto y la situación en que se encuentren, así como la composición del agua, la estructura del diamante o las medidas de la silla no dependen del conocimiento que un sujeto pueda tener de ellas. ¿No es absurdo, sin embargo, decir que el valor del vaso de agua en un bote salvavidas donde se encuentra un náufrago sediento es el mismo que el valor del que se encuentra sobre este escritorio? Del primero depende la vida de un hombre, del segundo depende muy poco.

Pero esto no es solamente absurdo, va además en contra de nuestra experiencia. Nunca encontramos como dato de la experiencia un valor constante. Al contrario, el valor es una de las cosas más variables que experimentamos. $\mathrm{Y}$ varía no solamente en grado o cantidad, sino también en calidad. Hace un rato me deleitaba la música, pero ahora me molesta porque estoy tratando de escribir. El diamante que le regalé a mi mujer cuando nos casa-

3 Hay que distinguir entre naturaleza concreta y naturaleza abstracta. La primera se refiere al singular, "este hombre", la segunda al universal, "el hombre". Mientras que la primera está limitada por todo el complejo circunstancial que particulariza al individuo, al segunda está limitada solamente por la definición de la esencia que la constituye. Así yo, Jorge J. E. Gracia, este hombre que escribe el presente ensayo, estoy limitado no solamente por el hecho de ser hombre, sino también como marido de mi mujer, padre de mis hijas, profesor de filosofía, etc. La naturaleza abstracta, por el contrario, se limita solamente, por la diferencia específica que limita su género, a esta clase de cosas. En la definición clásica aristotélica del hombre, por ejemplo, la naturaleza "hombre" se limita solamente por la racionalidad, que restringe su animalidad al subgrupo de animales humanos. 
mos ha adquirido un valor nuevo desde entonces como símbolo de nuestra relación matrimonial, un valor que se ahonda a medida que la relación se profundiza y solidifica con el tiempo y las experiencias comunes. Esta nueva dimensión axiológica del diamante sería inexistente para el ladrón que lo robara, o aun para mi esposa antes de que yo se lo hubiera dado como símbolo de nuestra unión.

Tanto en estos ejemplos como en todos los que podamos derivar de nuestra experiencia nos encontramos con que el valor del mismo objeto varía continuamente no sólo en términos cuantitativos dependientes de la oferta y la demanda, sino también cualitativos, dependientes de la situación y el sujeto que integran el todo axiológico en que vale.

La estructura de nuestra experiencia axiológica con un objeto que valoramos o que vemos que otros valoran es siempre la misma, aunque el valor y las circunstancias varien. En el caso de un objeto determinado $O$, presentado al Sujeto $S$ en tres ocasiones diferentes $\left(c, c^{\prime}, c^{\prime \prime}\right)$, esta estructura puede representar como:

1. $O$ tiene valor $y$ para $S$ en $c$.

2. $O$ tiene valor $v^{\prime}$ para $S$ en $c^{\prime}$.

3. $O$ tiene valor $v^{\prime \prime}$ para $S$ en $c^{\prime \prime}$.

En cada uno de estos casos, el valor de $O$ varía a pesar de que $O$ y $S$ son constantes. Es posible imaginar, sin dificultad, casos en que las circunstancias sean diferentes y que $O$ tenga el mismo valor para el mismo sujeto. Por ejemplo, la misma agua puede ser valiosa para calmar la sed en dos ocasiones diferentes siempre que no se haya consumido en la primera. Aquí se supone, sin embargo, que las circunstancias sean similares, o sea, que no haya otro vaso de agua más cerca del sujeto sediento, que el sujeto tenga sed, que el sujeto no sea paranoico y sospeche que alguien lo quiere envenenar, etc. En nuestra experiencia, el valor de un mismo objeto varía de acuerdo a las circunstancias y no se repite a no ser que la situación sea similar. Aun así, sin embargo, se puede notar que el sujeto es en sí variable y aun el objeto. El vaso de agua original se puede haber evaporado en parte, o tomado un sabor a vieja... El sujeto por otro tanto cambia continuamente. Para el niño, la sed, lo mismo que el deseo de ir al baño a desahogarse, son imperativos -bien lo sabemos los padres - mientras que para el adulto, el deseo es menos monopolizador de la atención, más distanciable, por lo que el valor del mismo objeto para el niño y el adulto, aun siendo éstos la misma persona, es diferente.

En nuestra experiencia, el valor siempre es particular, por lo cual nunca podemos anticipar con absoluta certeza el valor que las cosas puedan tener en un futuro. Sólo podemos anticipar con certeza la constante estructural del valor. La forma del caso futuro será: 


\section{4. $O$ tiene valor $x$ para $S$ en $n$.}

Pero el caso futuro es siempre hipotético y el valor permanece como una incógnita hasta que se ha actualizado; es una mera posibilidad, pero siempre una posibilidad particular, que no podemos anticipar por completo por nuestro desconocimiento de la situación circunstancial.

Esta mera posibilidad de valer, o sea, la dese-abilidad, es indiscutiblemente una característica del objeto, que depende de lo que el objeto es en sí y no de lo que el sujeto piense, sienta o desee, pero es precisamente por eso que no se puede identificar con el valor del objeto. No es ni $\boldsymbol{v}$, ni $\boldsymbol{v}^{\prime}$, ni $v^{\prime \prime}$, ni $x$, sino la suma de todos los valores posibles, $y$, por lo tanto, no es su valor. ${ }^{4}$

A esto puede responder el objetivista que estamos cayendo en una confusión total, ya que precisamente su tesis es que los valores particulares no constituyen el valor, y que, por lo tanto, el valor de un objeto lo constituye su deseabilidad. En el caso del vaso de agua, el valor no es el que tiene para el náufrago o para mí, sino todos los valores posibles que dependen de su deseabilidad intrínseca.

Esta distinción del objetivista se puede interpretar como que el valor $V$ de un objeto cualquiera es $a$ ) la suma de todos sus valores posibles $\left(V=v_{1}+\right.$ $\left.v_{2}+v_{3}+\ldots v_{n}\right)$ o, una segunda alternativa, $b$ ) que es superior a la suma de todos sus valores posibles $\left(V=v_{1}+v_{2}+v_{3}+\ldots v_{n}+x\right.$, donde $x$ es el valor excedente). Pero estas dos alternativas son igualmente inaceptables, pues si el valor de un objeto no es su valor particular en una situación determinada, hemos de concluir que no es solamente un valor totalmente desconocido, sino, además, incognoscible por el hombre, lo que hace contradictorio el hablar de él como valor, ya que sería sin duda algo que cae fuera de nuestro conocimiento y, por ende, de la especulación filosófica. ${ }^{5}$

Que el valor así interpretado no es conocido por el hombre, lo demuestra la experiencia. ¿Qué hombre puede vanagloriarse de conocer "el valor" $\left(v_{1}+v_{2}+v_{3}+\ldots v_{n}+x\right)$ ? Lo que encontramos en nuestra experiencia no

4 El valor no parece tener una definición sustantiva. Pero esto no quiere decir que cl valor sea indefinible o una cualidad objetiva, como dedujo G. E. Moore, o que consista en la aprobación, como concluyen los no-cognotivistas, sino solamente que la definición del valor ha de ser formal y no material. La definición que damos aquí no propone ser del mismo tipo ni estar por consiguiente en conflicto con definiciones normativas como las dadas por Robert S. Hartman, "el valor de una cosa es el cumplimientó de la definición de la cosa" (en varios lugares, por ejemplo, M. Bueno et al., La esencia del valor, México; UNAM, 1964, p. 77) o Francisco Romero, "el valor viene a ser la medida de la trascendencia, $y$, por lo tanto, de la efectiva realidad del ser" (Teoria del hombre, Buenos Aires, Losada, $3^{\text {a }}$ ed., 1965, p. 171), o como la que se da sobre el valor artístico en mi ensayo "Falsificación y valor artístico", Revista de Ideas Estéticas, 1972.

5 Este excesivo objetivismo no es poco común entre los axiólogos. Véase, por ejemplo, Max Scheler, Der Formalismus in der Ethik und die Materiale Wertethik, Bern, Francke, 1954, pp. $37 \cdot 8$. 
es "el valor", sino "este valor" $\left(v_{1}\right)$ o "aquel valor" $\left(v_{2}\right)$, valores particulares de un objeto determinado en una situación determinada, como ya dijimos. Tampoco encontramos en nuestra experiencia la suma de todos los valores posibles de un objeto $\left(v_{1}+v_{2}+v_{3}+\ldots v_{n}\right)$. Nuestras experiencias axiológicas de los objetos que nos rodean son sin duda magras.

El problema no termina ahí, sin embargo, pues no radica solamente en el hecho de que no conocemos "el valor" o "la suma de los valores posibles" de nada. El problema fundamental de esta doctrina es que es imposible llegar a conocer el valor si se interpreta de esta manera, ya que nuestras características espacio-temporales y biológicas ocluyen la experiencia de un valor absoluto. Un valor absoluto requiere un sujeto absoluto debido a que las circunstancias en que un objeto puede valer son infinitas. Pero ¿qué relevancia puede tener el valor absoluto de las cosas en un mundo de circunstancias? ¿No es absurdo decirle al náufrago de nuestro ejemplo anterior que el valor que él ve en el vaso de agua que le salvará la vida no es realmente el valor del vaso de agua en sí? La noción de un valor absoluto no es solamente contradictoria, sino que carece de importancia para los hombres.

La doctrina axiológica que propone el valor de un objeto como algo más que su valor particular $\left(v_{1} \circ v_{2} \circ v_{3} \circ \ldots v_{n}\right)$ y aun algo más que la suma de sus valores posibles $\left(v_{1}+v_{2}+v_{3}+\ldots v_{n}+x\right)$ carece de sentido. Supongamos que lo tenga por un momento y que, como nos dicen los partidarios de esta doctrina, el valor absoluto es la causa de los valores particulares, o sea, que las cosas valen particularmente porque valen absolutamente, ¿no podríamos preguntar cómo es posible que sepamos los valores particulares de las cosas aquí y ahora sin saber en qué consisten sus valores absolutos? ¿No habría que saber el valor absoluto antes de saber el particular? Pero el absoluto no lo sabemos, ni podemos saberlo. Entonces, ¿cómo conocemos o podemos conocer el particular? Si $v_{1}$ y $v_{2}$ y $v_{3}$ son los valores que son gracias a $V$, hemos de saber qué es $V$ para poder juzgar que el objeto $O$ tiene $v_{1}$ en $c$, $v_{2}$ en $c^{\prime}$ y $v_{3}$ en $c^{\prime \prime}$. En otras palabras, si el criterio que determina los valores particulares se deriva del valor absoluto, ¿qué criterio determina el valor absoluto de un objeto, y cómo, de facto, conocemos valores particulares sin conocer el absoluto? La única salida para el objetivista es negar, como hizo Platón, que los valores particulares sean realmente valores. Esto, sin embargo, no lo salva la contradicción en que cae al hablar del valor.

Esta doctrina incurre en el común error filosófico de aquellas teorías que con el propósito de explicar algo que es teóricamente difícil de explicar, destruyen la posibilidad de explicarlo, refugiándose en un criterio que trasciende la experiencia humana común; tratar de explicar el valor de esta manera no sólo destruye su criterio, sino, además, la posibilidad de encontrar alguno que tenga sentido para el hombre.

Aun suponiendo que estas dificultades se pudieran resolver haciendo 
referencia un ser absoluto, sujeto valorador del valor absoluto de un objeto, la hipótesis de un ser absoluto crea más problemas que los que resuelve, pues ¿cómo es posible que un objeto circunstancial y limitado tenga valor para un sujeto absoluto que, por consiguiente, no carece de nada? ¿Qué valor tendría, por ejemplo, nuestro vaso de agua para un sujeto que no ha tenido, no tiene o tendrá sed, porque no puede tenerla?

El valor existe sólo en el contexto carencia-tenencia que engendra el deseo en el sujeto carente por la tenencia del objeto. Es de la naturaleza del valor el ser efecto de una situación complementaria en que se une el sujeto finito, que, precisamente gracias a esa finitud, se agranda o completa por la contribución objetiva en la situación axiológiça con el objeto complementante, también finito. ${ }^{6}$ Es este desequilibrio ontológico entre sujeto y objeto que engendra el deseo en el sujeto por el objeto y que valoriza el objeto. Y es además en esta carencia-tenencia que se encuentran las razones del valor, como lo confirma la experiencia, pues no es hasta que una cosa se comprende, cuando puede ser deseada y valorada. El beduino que se encuentra con un automóvil en medio del desierto se morirá de hambre y sed a pesar de que el automóvil lo podría transportar al oasis salvador. Para él, carece, sin embargo, de valor, porque desconoce lo que es, su uso y posibilidades. Si fuera un camello la cosa sería diferente.

Un ser absoluto que no carece de nada no puede desear ni valorar. Para Dios, entonces, si es que Dios se concibe como un ser absoluto o con atributos absolutos, el mundo no vale ni puede valer, siendo sólo, quizá, objeto de una incomprensible compasión y misericordia; y, por supuesto, Dios tampoco vale para el mundo. Un ser absoluto valdría sólo para la nada, pero la nada no existe, lo cual nos lleva a nuestro punto de partida. ${ }^{7}$

Resumiendo, la contènción del objetivista que reduce la expresión " $x$ es bueno" o " $x$ tiene valor" a " $x$ es deseable" carece de fundamento, pues desvirtúa a la segunda aserción de su referencia potencial, reduciéndola a las

6 Este estado ontológico del sujeto es propiamente dicho una indigencia. El último término es un cultismo latino derivado de indigentia (pobreza, necesidad, miseria), usado frecuentemente por los escolásticos para describir el ser creado contingente en contraste con el ser del creador. Lo usa frecuentemente Guillermo de Auvernia en su Magisterium divinale (siglo xII, principios). Cicerón lo usa para referirse al deseo, la insaciabilidad; en Tusculanae disputationes 4, 9, 21: "indigentia est libido inexplicabilis", y un poco antes, 4, 7, 16: "indigentia, desiderium". Du Cange lo define como "quae (indigentiae) necessaria sunt ad vitam", citando el texto de la Crónica Fontanell apud Acher. t. 3. spicil. p. 227: "status sanctae Regulae periclitari coepit, ob inopiam videlicet compescendarum. Indigentiarum, quas B. Pater Benedictus in eadem lege monachis porrigendas proecepit". En el siglo XII se aplicaba frecuentemente a la materia, cf. Bernardo Silvestre.

7 Fsta imposibilidad y contradicción axiológica es uno de los argumentos más poderosos en contra de doctrinas filosóficas teístas tradicionales en que Dios se interpreta en la forma antedicha. Por esto es que el Motor Inmóvil de Aristóteles es totalmente ajeno al universo, pensamiento pensando pensamiento. Puede ser objeto del deseo porque no es un absoluto, estrictamente hablando. 
primeras, y añade a las primeras una dimensión de la que carecen. Este contraste entre actualidad y potencialidad, singularidad y pluralidad que las distingue se percibe claramente cuando se pregunta de cada expresión: ¿Qué valor? En el caso de las primeras la respuesta es simple: un valor económico, religioso, este valor de cambio, $\$ 5.00$, etc., pero en el caso de interpretar el valor como la deseabilidad, ¿qué responderíamos? Lo que es deseable o valorable no tiene obviamente un solo valor o "este valor", sino un valor múltiple e infinito y, se podría decir, un valor potencial, o sea, una mera posibilidad; de ahí la dificultad de contestar la pregunta.

En conclusión, el ser deseable no implica el valer, mientras que la aserción de valor requiere el haber sido deseado, aunque no se deduzca enteramente de ello.

Tampoco se puede identificar el valor, como afirman los subjetivistas, con el deseo, pues el ser deseado no implica necesariamente que lo deseado tenga valor en un momento dado o que sea valioso en la medida que sea deseado, pues podemos sin dificultad y con completo sentido preguntar, por ejemplo, si lo deseado tiene valor. ${ }^{8}$ De hecho, la mayoría de las discusiones éticas sustantivas giran alrededor de cuestiones de este tipo: "el adulterio es malo" contra "el adulterio es bueno", "el aborto es permisible" contra "el aborto nunca es permisible", a pesar de que todos están de acuerdo en que tanto el aborto como el adulterio son de facto deseados por muchos.

Un ejemplo concreto aclarará el asunto: en las elecciones puertorriqueñas para gobernar que se llevaron a cabo hace poco, tanto Hernández Colón, candidato del PDP, como Ferré, candidato del PNP, eran deseados por grandes segmentos de la población para ocupar el cargo de gobernador. Pero, obviamente, los dos no tienen el mismo valor. Precisamente ése es el núcleo de la cuestión electoral: cuál es el mejor, por ejemplo, el que vale más. Sería absurdo determinar este valor por el voto popular, pues aunque Hernández Colón ganó por un margen bastante alto, y aun si hubiera ganado con un porcentaje del $99.99 \%$ del voto popular o con el $100 \%$, demostrando con esto que era el candidato más deseado o el único deseado en términos cuantitativos para gobernador de la Isía, la cuestión de su valor no estaría determinada ni mucho menos por esa mayoría de deseadidad, como afirmarían los partidarios de Ferré o Ferré mismo en el caso que el último no tuviera ningún partidario. Todavía se podría hablar con todo sentido de Hernández Colón como un candidato peor que Ferré. Es más, aun la calidad del deseo es marginal a la determinación valorativa en cuestión, pues suponiendo que los partidarios de Hernández Colón estuvieran đispuestos a dar sus vidas si fuera nece-

8 Si así se hiciera, entonces no habría posibilidad de errar axiológicamente. Lo cual es absurdo. 
sario, cosa difícil de creer, para elegir a su candidato, mientras que los de Ferré no aceptaran ni los más mínimos sacrificios, aun así la cuestión del valor de los candidatos estaría indeterminada. El deseo de los votantes, cuantitativa o cualitativamente considerado, no le confiere valor al candidato, sólo lo elige o lo rechaza. Luego no es posible identificar el valor con el deseo ni aún siquiera con su intensidad.

Refiriéndonos de nuevo a nuestro ejemplo esquemático anterior, podríamos decir que a pesar de que los valores $v, v^{\prime}$ y $v^{\prime \prime}$ de $O$ son particulares y, por consiguiente, relativos a las circunstancias $c, c^{\prime}$ y $c^{\prime \prime}$ respectivamente en que $S$ se encuentra, con el resultado de que no son necesariamente vigentes para otro sujeto cualquiera, digamos $S^{\prime}$, son al mismo tiempo absolutos en virtud de la identidad del todo del cual son producto. Si, por ejemplo, establecemos que $S=S^{\prime}$, entonces los valores antedichos de $O\left(v, v^{\prime}\right.$ y $\left.v^{\prime \prime}\right)$ serán también valores para $S^{\prime}$, pudiendo sustituir en los tres casos $S^{\prime}$ por $S$ :
1. $O$ tiene valor $v$ para $S^{\prime}$ en $c$.
2. $O$ tiene valor $v^{\prime}$ para $S^{\prime}$ en $c^{\prime}$.
3. $O$ tiene valor $v^{\prime \prime}$ para $S^{\prime}$ en $c^{\prime \prime}$.

Si se aceptara, por el contrario, que el valor de $O$ es identificable con $O$ dependiente exclusivamente del ser deseado, cabe la posibilidad de que $O$ tuviera diferente valor para el mismo sujeto en situaciones idénticas, lo cual . supone una contradicción, ya que la variedad del valor en este caso sería inexplicable en términos racionales, o sea, las mismas razones justificarían valores diferentes. De manera que diríamos en el mismo caso " $x$ vale por $P$ " y " $x$ no vale por $P$ ". Si representamos " $x$ vale" por $Q y$, consecuentemente, " $x$ no vale" por $-Q$, podemos concluir que $P \supset Q \cdot-Q$, lo cual es absurdo. Por lo tanto, es precisamente la relatividad del valor lo que lo hace absoluto $y$, como resultado, no dependiente exclusivo del sujeto. ${ }^{9}$

La identificación que hace el subjetivista entre " $x$ es bueno" y " $x$ es deseado por $S$ " es, por consiguiente, tan errónea como la del objetivista, " $x$ es bueno" = " $x$ es deseable", pues mientras el objetivista descarta por completo el sujeto, el subjetivista reduce el valor a un acto subjetivo, distorsionando el propio sentido de la aserción axiológica. De ahí que se pierda su intencionalidad objetiva, " $x$ es deseado por $S$ " describe una situación particular en que el sujeto $S$ desea un determinado objeto $x$, mientras que la expresión " $x$ es bueno" es una aserción sobre $x$ y no sobre $S$, aunque sabemos, dada la naturaleza del lenguaje como artificio comunicativo, que detrás de " $x$ es bueno" hay un sujeto que dice la frase y, por lo tanto, que siempre que hay una frase " $x$ es bueno" hay otra o puede haber otra del tipo " $S$ dice:

9 Para una discusión del problema de la relatividad véase el texto de Stevenson citado en la nota 11. 
' $x$ es bueno'". ${ }^{10}$ Esto no implica, sin embargo, que " $x$ es bueno" sea equivalente a " $x$ es deseado por $S$ ", donde $S$ representa siempre un sujeto concreto, sino que " $S$ dice: ' $x$ es bueno'" es equivalente a " $x$ es deseado por $S$ ". En este caso se mantiene la forma lingüística indirecta sin distorsionar el sentido de la expresión, evitando el relativismo resultado de la identificación de " $x$ es bueno" con " $x$ es deseado por $S$ ". En virtud de esta identificación errónea, el relativista reduce el papel del axiólogo al del historiador de la situación, ya que traduce todos los juicios directos del tipo " $x$ es bueno" en aserciones indirectas del tipo " $x$ es deseado por $S$ ", excluyendo de su análisis toda aserción valorativa. ${ }^{11}$

Esta conversión errónea de " $x$ es bueno" a " $x$ es deseado por $S$ " cuando $S$ representa la primera persona $(S=$ yo), convierte al valor en algo enteramente personal y, como afirmaba Trasímaco, a la justicia en el interés del más fuerte. ${ }^{12}$ Obviamente, esta solución no es aceptable. ¿Quién de nosotros está dispuesto a abandonar sus derechos? Si la doctrina subjetivista fuera aceptada, no tendríamos bases teóricas para hacer ninguna reclamación en nuestro favor o en contra, pues el derecho y la justicia se convertirían, con todo valor, en actitudes puramente personales, lo cual quizá sea aceptable en teoría, pero ¿quién lo acepta en la práctica?13 El resultado del subjetivismo no es solamente una contradicción práotico-teórica, ${ }^{14}$ sino también la condo-

10 No se afirma con esto que el significado, la verdad, el sentido o la proposición '(P.Q) $\supset P^{\prime}$ no sea tautológica y, por lo tanto, atemporal. Sólo se afirma que "la expresión '(P.Q) $\supset \boldsymbol{P}^{\prime \prime}$ " implica, por definición, su posterioridad a su enunciador.

11 Este mundo ha sido expresado claramente por Charles L. Stevenson en Facts and Values; Studies in Ethical Analysis, New Haven and London, Yale Univ. Press, 3rd. ed., 1967, pp. 92-3: "But we must remember that the relativistic confusion, however curious it may seem to a so-called non-cognitivist, is nevertheless very tempting in the social sciences. A social scientist attempts to survey people's evaluations with a temporary detachment-to survey them without as yet taking sides, and thus without as yet participating in the normative issues that they may occassion. So his problem is basically different from the problem that he describes. His problem, in short, is concerned with what is considered good, whereas the problem that he describes is concerned with what is good. By an error parallel to the one that William James called 'the psychologist's fallacy', however, he may suppose that his problem is not basically different from the one that he describes. And when he yields to this tempting error he may wander from 'considered good' to 'good' without realizing that he is is doing so."

12 Platón, La República I, 338C; en la ed. de Loeb Classical Library, 1969, p. 47.

13 Stevenson propone su versión de este argumento de la siguiente manera (op. cit., p. 92): "Indeed, the confusion is such a thorough one that it would be impossible to live by relativism. A consistent rclativist, when asked what is good or right, etc., would in effect discuss only what is or was considered good, right, etc., and thus would himself stand committed to no value judgments whatsoever. He would be a nonparticipant on evaluative issues - as no man, in practice, can be."

14 Uno de los casos más extraordinarios de la conciencia de esta contradicción lo cons. tituye B. Russell: "Se me acusa, quizá con razón, de caer en una contradicción porque, si bien sostengo que las valoraciones éticas son subjetivas, me permito emitir opiniones categóricas sobre cuestiones éticas. Si hay en ello alguna contradicción es de una naturaleza tal que no puedo librarme de ella sin caer en la insinceridad. Más aun, un sistema con. tradictorio puede muy bien contener menos falsedad que uno coherente... No estoy dis- 
nación del individualismo absoluto y, como diría Hobbes, de un estado de guerra caótico en que cada uno se defiende como puede, aniquilando y destruyendo a su semejante en la medida que le sea posible. ${ }^{15}$

Por el contrario, si se mantiene la distinción, como he sugerido, entre " $x$ es bueno" y " $x$ es deseado por $S$ " o, lo que es lo mismo, " $S$ dice: ' $x$ es bueno'", es evidente que el axiólogo no está condenado a ser un mero historiador, pues sea cual fuere el sujeto $S$, incluyendo por supuesto al axiólogo mismo, puede mantener la irreductibilidad entre sus aserciones valorativas sobre un objeto y el acto personal de aseveración de dichas aserciones, además de poder dar, como resultado, las razones por las cuales se afirma lo que dice. De manera que la consideración del sujeto no condena al subjetivismo relativista excepto cuando se identifica el valor con el acto subjetivo de desear.

Como diría Russell, llamamos a algo 'bueno' cuando lo deseamos, aunque hemos de añadir que no exclusivamente porque lo deseamos.

\section{III}

El valor no es una cualidad absoluta sino relacional, o sea, una cualidad que existe solamente en virtud de varios factores relacionados entre sí o, como propone Frondizi, una cualidad Gestalt, de un todo. ${ }^{16}$ Hay que tener cuidado con esta expresión, sin embargo, pues el valor no es una cualidad del todo que forman el sujeto y objeto en su circunstancia o situación particular. El valor es el resultado del todo. Sus causas son los elementos que forman el todo (miembros de la estructura), sin los cuales, en su organización o estructura particular como parte del todo, no habría tal cualidad de valor, pero al mismo tiempo el valor es una cualidad del objeto, no del todo, o sea, una cualidad del miembro u objeto valorado, aunque causada por los miembros organizados en un todo determinado. En este sentido podemos decir que el valor es una cualidad estructural, pero no de la misma especie, por ejemplo,

puesto a renunciar al derecho de sentir y expresar pasiones éticas; ninguna cantidad lógica, ni siquiera de mi propia lógica, podrá persuadirme de que debo hacer tal renuncia. Hay hombres a quienes admiro y otros a quienes considero despreciables; algunos sistemas políticos me parecen tolerables, otros detestables. El placer ante el espectáculo de la crueldad me horroriza y no me siento avergonzado por ello. No estoy más dispuesto a renunciar a todo esto de lo que estoy a renunciar a la tabla de multiplicar." Lo que se le pide a Russell no es que renuncie a la tabla de multiplicar o a sus sentimientos y convicciones éticas; lo que se le pide es que renuncie a su teoría sobre éstas. Utilizo la traducción del pasaje que da Frondizi en $¿ Q u e ́$ son los valores? Introducción a la axiologia, México, Fon-

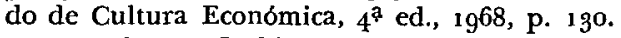

15 Thomas Hobbes, Leviathan I, cap. 13. New York, Library of Liberal Arts., 1958, p. 107 . No quiero decir con esto que Hobbes esté en contra del subjetivismo, pues bien se sabe que su teoría ética es subjetivista. Lo que quiero decir es que Hobbes reconoce a dónde lo lleva su subjetivismo -al estado de guerra caótico que describe en el pasaje aludido - y que por esto se ve forzado a dar la solución que da a la situación miserable en que según él se encuentra el hombre, o sea, el monstruo tiránico Leviatán.

16 Frondizi, p. 160. 
que la cualidad de la romboidad que poseen cuatro puntos cuando se organizan en forma de romboide $\because{ }^{17}$, La romboidad de los cuatro puntos es una cualidad del todo que forman los cuatro puntos y no de uno de ellos, mientras que el valor no es una cualidad del todo valorante que forman sujeto robjeto circunstancia sino una cualidad del objeto dentro del todo. Es el resultado de una proyección del deseo del sujeto por el objeto deseable en circunstancias propicias y, por lo tanto, una cualidad proyectada por el sujeto deseante y adquirida por el objeto deseable en la situación particular en que es deseado. Cuando decimos " $x$ tiene valor" $o$ " $x$ vale" no estamos predicando la cualidad del valor del todo formado por el sujeto y el objeto valorado, sino solamente de ese objeto deseable; hay, por lo tanto, una polarización entre objeto y sujeto dentro del fenómeno valorativo, producto en parte de la intención del sujeto.

Se ha de advertir, además, que no toda estructura produce valor. Las flores de un búcaro forman un ramo, pero carecen de valor si no está presente el sujeto que las desee en la situación particular. De modo semejante, nuestro rombo formado por los puntos tiene una característica estructural de romboidad, pero no tiene valor. Esto se debe a que los miembros de la estructura que da lugar al valor son constantes: el sujeto valorador, el objeto valor-able y las circunstancias concretas en que se encuentran. Sin embargo, tanto el objeto como el sujeto y las circunstancias, en sí subestructuras, son variables. No es "este hombre", ni siquiera "el hombre", el elemento necesario en el todo valorante, ${ }^{18}$ no es "este objeto", ni "esta clase de objetos" (materiales, ideales, esenciales, espirituales, etc.), los que son valorables $y$, por lo tanto, imprescindibles para la presencia del valor, sino todo lo que existe simplemente porque existe y que, por ende, puede ser objetivado por la conciencia del sujeto; y tampoco son "estas circunstancias" o "aquellas", sino cualquiera situación concreta propicia en que se encuentren el objeto y sujeto determinados las que dan lugar al valor. El valor depende siempre de la tríada valorante, aunque, obviamente, la existencia de la tríada valorante en un caso particular no asegura la presencia del valor.

El sujeto deseante, el objeto dese-able y las circunstancias propicias al valor y al deseo son las causas del valor, constituyendo la situación ontológica que lo produce. Es la deseabilidad la que provee las posibilidades valo-

17 Estoy consciente de que la homogeneidad de los puntos-miembros de la estructurarombo hace inapropiado el ejemplo, ya que quizá sugiera que en el todo estructural los miembros han de ser homogéneos mientras que su característica esencial es precisamente lo contrario. Hecha esta aclaración creo que el ejemplo es válido en el contexto en que se da.

18 Los medievales hablaban de ángeles en el contexto axiológico y nosotros podríamos hablar de marcianos. Es posible que el hombre sea el único ser valorador en la tierra, pero esto no invalida la posibilidad de lo contrario en el futuro, o fuera de nuestro planeta. 
rativas, gracias a las limitaciones impuestas a la esencia del objeto por su existencia concreta; mientras que el deseo realiza la dimensión axiológica del objeto, apremiado por las condiciones circunstanciales y la pre-visión del valor.

La causa del valor, entonces, no es una, sino múltiple. El valor es el resultado de cierta estructura situacional entre sujeto, objeto y circunstancia, $\mathrm{y}$, por lo tanto, depende directamente de la estructura considerada como un todo, pero, precisamente por depender de la estructura, depende indirectamente de los miembros que forman esa estructura. De manera que el valor depende tanto de la estructura total que forma la estructura valorante $\left(S_{\mathbf{C}} \mathrm{O}\right)$ como de la naturaleza concreta de los miembros de dicha estructura,

pues son precisamente los miembros de la estructura valorante determinada que, gracias a las características resultado de su condición ontológica, pueden ser miembros de dicho todo.

En el caso anterior del rombo, la romboidad, como cualidad, aparece no solamente causada por la forma estructural que toman los cuatro puntos, sino también por esos puntos concretos que, por ser lo que concretamente son y como lo son, poseen la posibilidad de formar parte de una figura romboide, como también de un número infinito de figuras geométricas. Del mismo modo, el valor estético del ramo de flores es causado tanto por la estructura $S \underset{\mathrm{C}}{+} \mathrm{O}$ particular como por la subestructura particular del objeto, o sea, el

ramo de flores. Además, este valor es causado a su vez por estas estructura y subestructura consideradas como todos de sus miembros, ya que la estructura en sí no es más que la actualización ontológica de una de las posibilidades inherentes en la naturaleza concreta de cada una de las flores que forman la subestructura del ramo dentro de la estructura valorante. Esto no quiere decir que las características del todo sean la suma de las características de los miembros o que se puedan reducir a las características de los miembros, sino que el todo es el resultado de las posibilidades inherentes a los miembros del todo. Por sí mismos, los miembros no son el ramo particular de que hablamos, ya que las flores, aunque juntas, pueden estar desarregladas y, por lo tanto, no resultar en un valor estético; pero esas flores, por ser lo que son individualmente, pueden llegar a formar un todo particular, un ramo que el sujeto encuentre bello. La belleza de ese ramo depende tanto de la estructura particular que toma la tríada valorante considerada como un todo como de las naturalezas concretas del ramo, de las flores y del sujeto particulares. ${ }^{19}$

El valor es, pues, como dije al principio de este ensayo, la cualidad que

19 Quizá la traducción analógica de este análisis causal a términos tradicionales como, por ejemplo los aristotélicos, ayude a su comprensión. Podríamos decir, entonces, que la deseabilidad es similar a la causa formal aristotélica, el deseo subjetivo a la causa eficiente, las circunstancias a la causa material y el valor a la causa final de esta cualidad. 
adquiere lo deseable cuando es deseado y, como tal, basado en la situación ontológica particular de la cual proviene y que relaciona los miembros de la estructura que lo produce.

\section{IV}

Qué podemos responder, entonces, a la pregunta: ¿Son las cosas deseadas porque tienen valor o tienen valor porque tienen valor o tienen valor porque son deseadas?

Si nuestra argumentación en las secciones I-III de este ensayo es correcta, no podremos contestar con las consabidas respuestas objetivista (las cosas son deseadas porque valen) o subjetivista (las cosas valen porque son deseadas). La primera respuesta confunde el producto con la causa, mientras que la segunda reduce la pluralidad causal axiológica a una sola causa. La única alternativa es contestar, de acuerdo con nuestra definición, que las cosas tienen valor porque siendo dese-ables son deseadas en circunstancias particulares. Las cosas no son por consiguiente, deseadas porque tienen valor, sino que el valor es la cualidad que adquieren gracias en parte al deseo. El subjetivista, entonces, se lleva una buena tajada del melón, pues acepta la posterioridad del valor a la valoración, aunque no se lo lleva entero por el error que comete al reducir el valor a su causa subjetiva. El objetivista, por el contrario, confunde el orden causal, concluyendo erróneamente que el valor es anterior a la valoración.

La perspectiva, subjetivista, que reduce las causas determinantes del valor a una, el deseo, nace, en primer lugar, de un escepticismo básico sobre la posibilidad de concordar en los valores. Partiendo de dos premisas: a) el desacuerdo que existe en cuanto a los valores y $b$ ) la necesidad explanatoria de encontrar una causa a tales valores, busca resolver el problema reduciendo el valor al deseo. Necesariamente, si el valor varía de sujeto a sujeto irrespectivamente del objeto, irrespectivamente del sujeto, el acuerdo axiológico sería fácil y una realidad evidente dondequiera. El hecho es que no es así. $\mathrm{Ni}$ los filósofos más objetivos y cuidadosos llegan a un acuerdo sobre el valor aun en los casos más sencillos. ¿Cómo podemos, entonces, poner la causa del valor en el objeto? Hacerlo así supone no prestar atención a la realidad empírica que confrontamos a diario y de la que hemos de partir si es que nuestras teorías intentan tener alguna relación con el mundo que nos rodea. El análisis de esa rcalidad nos lleva a una conclusión solamente: si el valor es variable, la variedad tiene que entrar en su causa esencial, lo que explica por qué ha de ser en el sujeto y no en el objeto que se ponga.

Este razonamiento del subjetivista contiene dos falacias: primera, la generalización de un escepticismo empírico; ya que no hay concordancia axiológica sustancial, se concluye que no puede haber, o sea, de que el valor del mismo objeto $O$ se dé como $P$ por un sujeto y como $Q$ por otro, se concluye 
que un objeto cualquiera $x$ no puede tener el mismo valor para varios $y$, menos aun, para todos los sujetos. La falacia es obvia, ya que no sólo llega el subjetivista a una generalización infundada, sino que viola sus propios principios empíricos, contradiciéndose inocentemente.

Aún más desastroso que esta generalización contradictoria, es el error capital que comete el subjetivista al omitir en el análisis de la causalidad esencial axiológica un factor importantísimo que nos presenta la experiencia: la circunstancia o situación, pues es allí precisamente que el factor variedad se introduce más significativamente entre las causas del valor. La situación, en última instancia, como he dicho arriba, es el factor que completa y, en cierto sentido, determina la relación de los miembros en la tríada causal que produce el valor. La variedad factual del valor, entonces, no es necesariamente el resultado del sujeto, sino de la estructura valorante $\begin{aligned} & \text { sujeto tobjeto } \\ & \text { circunstancia, que }\end{aligned}$ toma su particularidad precisamente de la situación única que representa la unión de sus miembros.

En segundo lugar, la reducción subjetivista es producto de una confusión común en la filosofía contemporánea entre criterio y esencia. Un ejemplo clásico es el que comete Perry al identificar el valor de un objeto con el interés que tiene el sujeto en tal objeto: "That which is an object of interest is eo ipso invested with value. Any object, whatsoever it be, acquires value when any interest, whatever it be, is taken in it; just as anything whatsoever becomes a target when anyone whosoever aims at it", 0 sea, " $x$ is valuable $=$ interest is taken in $x^{\prime \prime} .{ }^{20}$ Lo que lleva a Perry a identificar el valor con el interés o la actitud subjetiva es una de las causas imprescindibles del valor: para que haya valor, ha de haber interés. Cion esto estamos de acuerdo, siempre que cambiemos la palabra 'interés' por 'deseo'. Con lo que no estamos de acuerdo es con la reducción del valor al interés. El interés, o el deseo en nuestra terminología, es un elemento que siempre acompaña al valor, pero no es el único elemento causal del valor; por ser un elemento imprescindible o constante estructural del valor representa un factor criteriológico negativo infalible: si no hay deseo, no hay valor. El valor, sin embargo, es más que el deseo. Su esencia no incluye solamente el deseo, sino también la deseabilidad (y la situación). Lo que define Perry no es el valor, sino un criterio del valor, mientras que la esencia del valor tiene que estar dada por una definición mucho más compleja y determinante que la que él da.

La confusión objetivista que anticipa el valor al sujeto, desconectando así el nexo causal del valor con el deseo subjetivo, surge de la aprehensión ante las consecuencias de incluir como causa del valor una variable subjetiva. Si el deseo subjetivo es causa del valor, hay que abandonar la más mínima es-

20 R. B. Perry, General Theory of Value, Cambridge, Mass., Harvard Univ. Press, 1950, pp: $115^{-16 .}$ 
peranza de encontrar valores absolutos y, por lo tanto, la sociedad humana cae sin remedio en el más profundo caos axiológico. Es imprescindible, por lo tanto, purgar lo variable del valor, haciéndolo independiente del sujeto.

Esta confusión del objetivista se agudiza en parte gracias a una comparación comúnmente hecha entre los pares "percepción-objeto percibido" y "valoración-valor". ${ }^{21}$ Se dice que como la percepción requiere la anterioridad del objeto, así la valoración requiere la anterioridad del valor. Pero la proporción así establecida es errónea, pues, en primer término, lo que se valora en la valoración no es el valor; el valor no puede valorarse ya que es el resultado de la valoración. Lo que se valora es el objeto valor-able, así como lo que se percibe es el objeto percept-ible. Tanto el objeto de percepción como el objeto de valoración anteceden a los actos de percepción y valoración respectivamente.

En segundo lugar, la proporción correcta no es entre "percepción-objeto percibido" y "valoración-valor", sino entre "deseo-deseable-valor" y "conocimiento-cognoscible-verdad". La semejanza entre el valor y la verdad es sorprendente. ${ }^{22}$ Tanto la verdad como el valor son cualidades estructurales resultado de la tríada $\begin{aligned} & \text { sujeto robjeto } \\ & \text { circunstancia. }\end{aligned}$ de conocer aprehende el objeto cognoscible que, cristalizando en el juicio, adquiere la cualidad de ser verdadero. $L a$ verdad, entonces, reside ontológicamente en el juicio subjetivo cuando éste, " $x$ es blanco", por ejemplo, corresponde a un estado actual de hechos: $x$ es blanco. Por consiguiente, la verdad es posterior, tanto al acto de conocer como al objeto cognoscible y, propiamente hablando, se conocen los objetos verdadera o falsamente, no se conoce la verdad. La verdad es sólo la cualidad que tiene el producto del conocimiento fidedigno. ${ }^{23}$

De modo semejante, con respecto al valor, en el acto subjetivo axiológico se desea el objeto deseable, relación que actualiza el valor, una cualidad en el objeto. Por consiguiente, propiamente hablando, no se valora el valor,

21 Véase, por ejemplo, Frondizi, p. 25 .

22 Tanta es la semejanza, que da origen en la Edad Media a la doctrina de los trascendentales, en que la verdad se hace convertible con el bien, aunque esta convertibilidad se interpreta generalmente como una convertibilidad del supósito. Véase, por ejemplo, mi discusión del problema en el artículo "The Convertibility of Ens and Unum According to Guido Terrena", Fran. Stds., 1972.

23 Esto no quiere decir que no podamos decir que "conocemos la verdad", sino solamente que este uso de 'verdad' no es el mismo que tiene el término cuando se dice de un juicio que es "la verdad". Y además que el segundo deriva su significado del primero. Esta confusión es particularmente aguda en pensadores neo-platónicos medievales como Agustín y Anselmo, que llegan hasta el punto de confundir la realidad ontológica del objeto con la verdad y con su valor. Véase, por ejemplo, el Dialogus de veritate de Anselmo, cap. 10, en que Dios es identificado con la verdad, o en el cap. 7 , en el cual se dice que "la verdad está... en la esencia de las cosas existentes, pues son lo que son en la verdad suprema". $P L 15^{8}, 47$. No he podido cotejar el texto de Migne con la edición crítica de Schmitt. 
sino el objeto deseable cuando se desea, aunque, impropiamente, tal como "se conoce la verdad", "se puede valorar el valor". El lenguaje impropio, sin embargo, debe ser expurgado de la filosofía en cuanto sea posible.

Para terminar esta sección y con ella poner fin al ensayo, debo llamar la atención sobre un punto importante que ha salido a relucir en esta última parte, a saber, que las razones propulsoras del subjetivismo y objetivismo son, aunque parezca sorprendente, ajenas y externas al análisis del problema causal axiológico. Tanto el objetivista como el subjetivista deducen la causa del valor y su relación con el objeto, sujeto o circunstancia en base a supuestos previos o consecuencias que ellos creen están implícitas en sus respectivas teorías, sin prestar atención preeminente al análisis del fenómeno axiológico mismo. Aunque este procedimiento es sin duda una parte integral y necesaria tanto del proceso filosófico como del científico, lo primero que hay que hacer en toda investigación, como han aprendido los científicos, pero los filósofos insisten en olvidar, es prestarle atención al fenómeno mismo que se ha de investigar y más tarde a las implicaciones y consecuencias de las hipótesis explicativas que proponen esclarecerlo. De otra forma, tendremos que concordar con el subjetivista en que no hay concordancia posible.

Department of Philosophy

Jorge J. E. Gracia

BUfFaLO UNIVERSITY 